\title{
Studierende auf den Einsatz von maschinellem Lernen vorbereiten
}

\author{
Raphaël Bonvin ${ }^{a}$, Joachim Buhmann ${ }^{b}$, Carlos Cotrini Jimenez ${ }^{c}$, Marcel Eggerd, Alexander Geissler ${ }^{e}$, Michael \\ Krauthammer ${ }^{f}$, Christian Schirlog, Christiane Spiess ${ }^{h}$, Johann Steurer ${ }^{f}$, Kerstin Noëlle Vokingeri, Julia Vogt ${ }^{b}$ \\ a Prof. Dr. med., Universität Fribourg; ${ }^{b}$ Prof. Dr., ETH Zürich; ${ }^{c}$ Dr., PhD, ETH; d Prof. Dr. med., Universität Bern; e Prof. Dr., Universität St. Gallen; \\ ${ }^{f}$ Prof. Dr. med., Universität Zürich; ${ }^{9}$ Dr. med., Universität Luzern; ${ }^{h}$ Dr., Universität Basel; ${ }^{i}$ Prof. Dr. iur. et Dr. med., Universität Zürich
}

Die Digitalisierung hat die Medizin bereits verändert und wird die ärztliche Tätigkeit auch in Zukunft stark beeinflussen. Es ist deshalb wichtig, dass sich angehende Ärztinnen und Ärzte bereits während des Studiums mit den Methoden und Einsatzmöglichkeiten des maschinellen Lernens auseinandersetzen. Die Arbeitsgruppe «Digitalisierung der Medizin» hat dazu Lernziele erarbeitet.

In den nächsten Jahren werden Methoden des maschinellen Lernens immer häufiger in der Medizin eingesetzt werden. Ärztinnen und Ärzte haben jetzt die Chance, diesen Umbruch ihres Berufs aktiv mitzugestalten und die neuen Technologien insbesondere für die Interaktion "Arzt-Patient» zu nutzen und in die Patientenberatung zu integrieren.

Dazu benötigen sie ein Grundverständnis der entsprechenden Technologien, um sich an diesem Prozess aktiv beteiligen zu können und die neuen Technologien in der ärztlichen Praxis bewerten und sinnvoll einsetzen zu können. Es ist deshalb wichtig, das maschinelle Lernen bereits im Studium zu behandeln.

\section{Einsatzmöglichkeiten der Digitalisierung}

In der Arbeitsgruppe «Digitalisierung der Medizin» des Bildungsnetzwerkes Medizin haben wir die Digitalisierung der Medizin und ihre Implikationen insbesondere durch das maschinelle Lernen diskutiert. Zudem haben wir erörtert, welche Bildungsinhalte Studierenden im Laufe des Medizinstudiums vermittelt werden sollten. Wir sind uns bewusst, dass wir mit diesem Fokus auf maschinelles Lernen nur einen Teil der Digitalisierung abdecken. Andere Einsatzmöglichkeiten der Digitalisierung sind beispielsweise die Telemedizin, die kontinuierliche Erfassung physiologischer Parameter (wie Puls oder Blutdruck) und die Beratung und Betreuung von Patientinnen und Patienten mit chronischen Krankheiten mittels Apps.

\section{Maschinelles Lernen im Studium}

Konkret setzte sich die Arbeitsgruppe mit der zentralen Frage auseinander: Was sollten alle Medizinstudierende am Ende ihres Studiums über "machine learning» bzw. maschinelles Lernen verstehen, wissen und anwenden können? Zukünftige Medizinerinnen und Mediziner sollten in der Lage sein, ein System des maschinellen Lernens und dessen Anwendung in der jeweiligen klinischen Situation anhand von Kriterien zu validieren. Spezifisch sollten sie befähigt sein, mittels dieser Methoden generierte Ergebnisse korrekt zu deuten und anschliessend im klinischen Kontext anzuwenden.

Deshalb schlagen wir die folgenden generellen Lernziele zum Thema maschinelles Lernen vor: Basiswissen über Daten und Datenverwaltung, Grundwissen zu Konzepten, Validierung von Ergebnissen, die Ärztinnen und Ärzte mit maschinellem Lernen erzeugen, Wissen über die Anwendung in der medizinischen Praxis und Kenntnisse über rechtliche und ethische Aspekte der Anwendung in der Praxis. Für Studierende, die sich ein vertieftes Wissen aneignen wollen, schlagen wir vor, spezielle Kurse anzubieten.

\section{Grundkenntnisse über Daten}

Voraussetzung für die Entwicklung von Algorithmen und die Anwendung der Algorithmen in der Praxis sind Daten. Diese Daten müssen nach bestimmten Standards gespeichert werden, um für die Weiterverarbeitung nutzbar zu sein. Obwohl Algorithmen zu- 


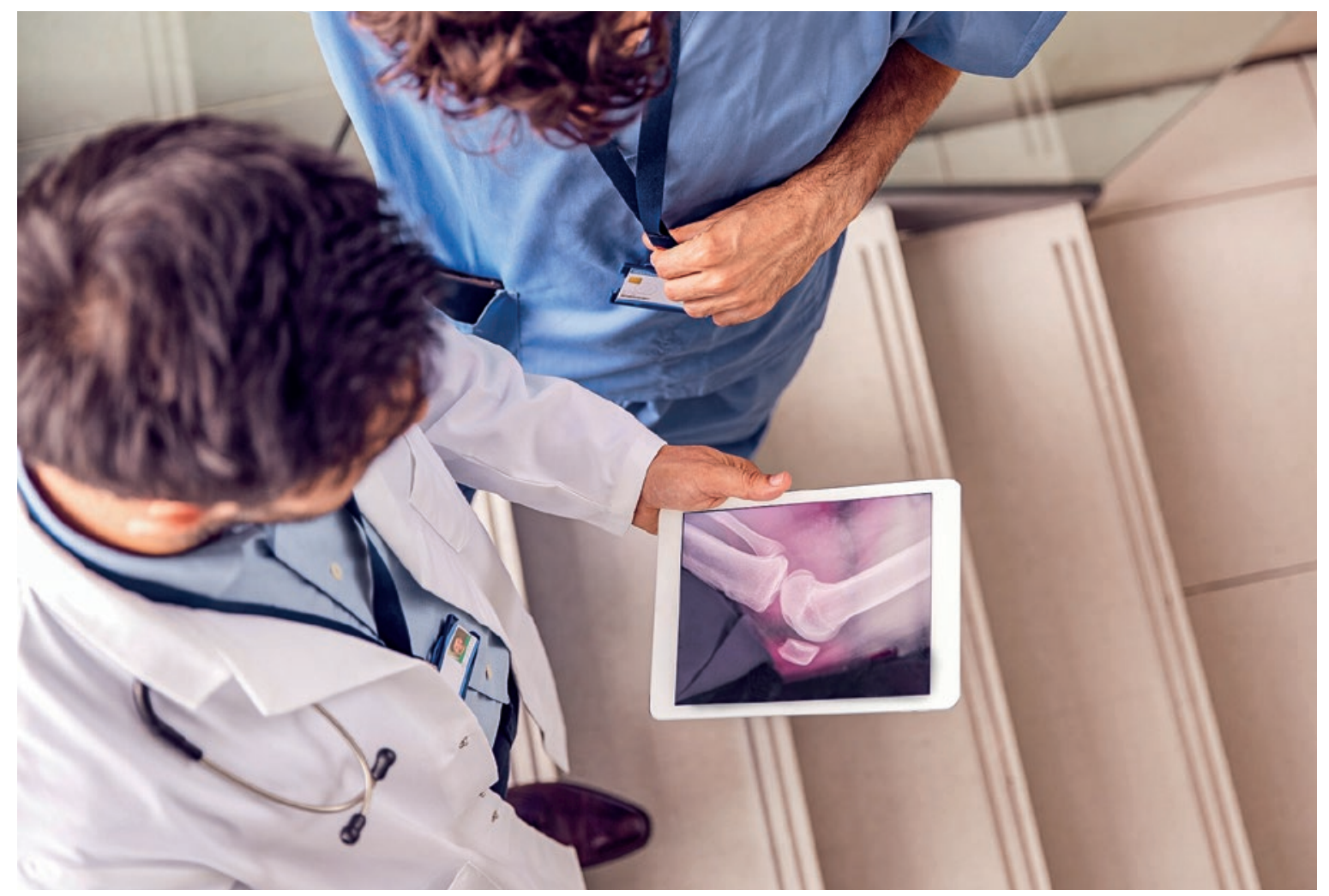

Es ist wichtig, dass Ärztinnen und Ärzte sich frühzeitig mit der Digitalisierung auseinandersetzen. So können sie kommende Veränderungen in ihrem Beruf aktiv mitgestalten.

nehmend unstrukturierte Daten (Bilder, Texte) verarbeiten können, sind sie weiterhin auf strukturierte (kodierte) Daten, wie etwa präzise Diagnosen oder eine detaillierte Dokumentation der Therapieverläufe, angewiesen. Strukturierte Daten sollten national und international über Standards harmonisiert erhoben werden, damit Daten aus verschiedenen Quellen (Spitälern, Arztpraxen) für das Trainieren von Algorithmen zusammengelegt werden können.

- Grundkenntnisse zu Datenstandards sollten gelehrt werden. Das gilt sowohl für Standards zur Übermittlung von unstrukturierten Daten wie auch zur Kodierung von Krankheiten mittels internationaler Standards wie ICD-10-Codes oder SNOMED Clinical Terms.

- Ein Verständnis für die verschiedenen Formen von Datenintegration (virtuell, materialisiert), aber auch für neue Formen von verteiltem maschinellem Lernen (föderiertes Lernen) sollte als Grundkonzept vorhanden sein.

\section{Grundkenntnisse der Konzepte}

Die Studierenden sollen verstehen, was ein Modell ist, was modellieren heisst, und wissen, was Expertinnen und Experten des maschinellen Lernens unter einem
Algorithmus verstehen. Wichtig ist, dass die Studierenden die grundlegenden Konzepte von maschinellem Lernen kennen und verstehen. Ein vertieftes Wissen über sich kontinuierlich entwickelnde technische Details ist nicht notwendig. Ein Grundwissen in Programmierung und Statistik ist unverzichtbar, um Einsichten darüber zu gewinnen, wie statistische Modelle im Rahmen von maschinellem Lernen trainiert werden. Mit Hilfe dieser Kenntnisse können Medizinstudierende feststellen, ob ein Modell für eine bestimmte Fragestellung geeignet ist oder nicht.

Einige Themen, die zu diesen Grundlagen gehören, sind:

- Wahrscheinlichkeiten und Erwartungswerte

- Grundlegende Sätze der Statistik wie z.B. das Gesetz der grossen Zahlen

- Lineare und logistische Regressionsmethoden

- Modelltraining, Überanpassung («overfitting») und Unteranpassung («underfitting»)

- Lernen von Modellparametern

- Konzepte neuronaler Netzwerke

Wenn immer möglich, sollen diese Themen anwendungsbezogen und anhand von Praxisbeispielen gelehrt werden. Grundlegende Kenntnisse des Programmierens müssten vorausgesetzt oder aber zusammen mit der Anwendung von Bibliotheken für maschinelles 
Lernen wie scikit-learn und Keras (beide für die Programmiersprache Python) im Unterricht vermittelt werden.

\section{Validierung von Ergebnissen}

Aus konzeptioneller wie auch aus klinischer Sicht stellt sich die Validierung der Ergebnisse, die mit maschinellem Lernen generiert werden, als eine prioritäre Aufgabe dar. Die Studierenden sollen die Frage, ob sie den Ergebnissen vertrauen dürfen, beantworten können.

Einige Themen, die zu diesen Grundlagen gehören, sind:

- Verfahren zur Kreuzvalidierung

- Beurteilung binärer Klassifikatoren: Sensitivität, Spezifität, AUC («area under the curve»)

- Extrapolierbarkeit von Ergebnissen eines Algorithmus, der an einer Kohorte entwickelt wurde, auf Personen einer anderen Kohorte

- Zudem sollten die Studierenden fähig sein, die Robustheit und eine mögliche Voreingenommenheit (Bias) von Systemen mit Künstlicher Intelligenz zu erkennen und Anpassungen, so etwa das Trainieren mit neuen Daten, zu begleiten.

\section{Einsatz in der klinischen Tätigkeit}

Maschinelles Lernen kann autonom und nicht-autonom (als Entscheidungsunterstützung) eingesetzt werden. Das bedeutet:

- Ärztliches Personal muss befähigt sein, die Rolle von maschinellem Lernen im Gesamtprozess der Behandlung zu verstehen.

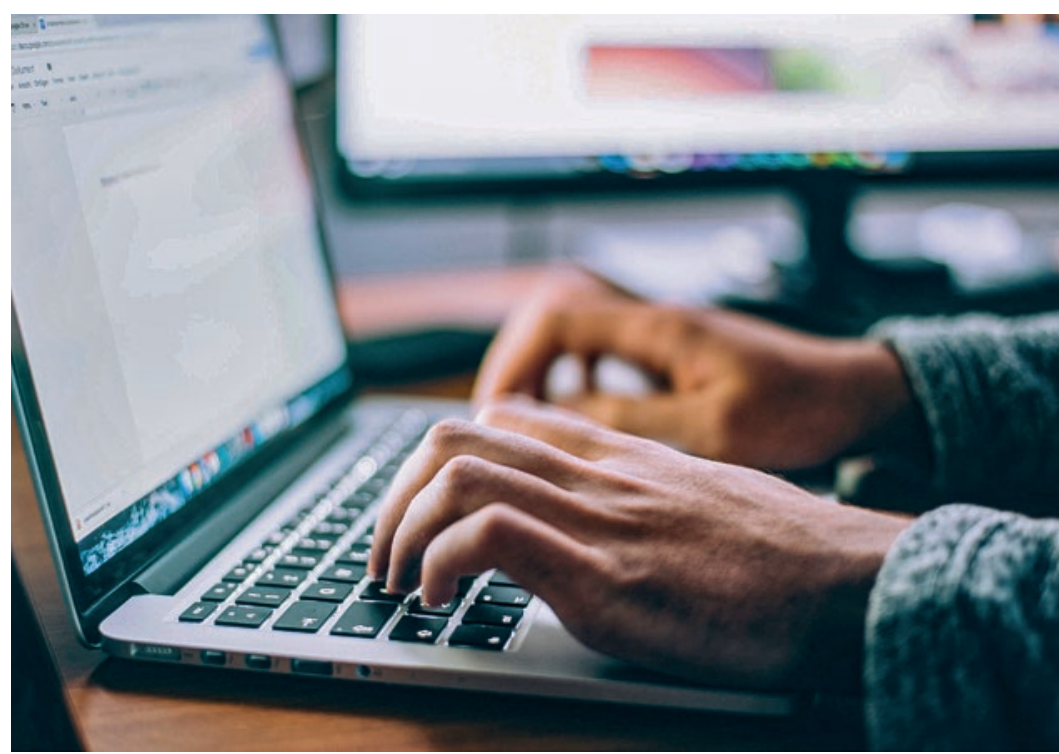

Medizinstudierende wünschen sich, dass der Einsatz digitaler Methoden im Studium stärker thematisiert wird.
- Die Medizinstudierenden müssen imstande sein, den Patientinnen und Patienten die Ergebnisse des Modells verständlich zu erläutern.

\section{Ethische und rechtliche Fragen}

Das maschinelle Lernen wirft neue ethische und rechtliche Fragen auf. Studierende müssen für diese gesellschaftspolitischen Aspekte sensibilisiert werden und müssen in diesen für das Berufsbild wesentlichen Fragen Stellung beziehen können. Hierzu einige Beispiele:

- Ethische und rechtliche Anforderungen an den Umgang mit Daten: Was sind die Voraussetzungen, damit (Patienten-)Daten erhoben und weiterverwendet werden dürfen? Was für Besonderheiten gibt es bei urteilsunfähigen oder verstorbenen Patientinnen und Patienten?

- Ethische und rechtliche Anforderungen an die Datensicherheit: Was für Voraussetzungen gibt es z.B. in Bezug auf die Speicherung oder Anonymisierung von Daten?

- Ethische und rechtliche Anforderungen an die Entwicklung und den Einsatz eines MaschinellenLernen-Modells: Wie transparent und nachvollziehbar werden algorithmische Entscheidungen getroffen? Könnten solche Systeme anhand der Lerndaten voreingenommen sein? Wie können solche Systeme fortlaufend mit neuen Daten angepasst und verbessert werden? Welche Regeln der Kunst sind bei Methoden des maschinellen Lernens zu beachten, und können die Entscheidungen von Maschinellen-Lernen-Systemen kausal begründet werden?

- Rechtliche Möglichkeiten und Grenzen beim klinischen Einsatz dieser Methoden: Welche medizinischen Tätigkeiten und Entscheidungen dürfen von Maschinen übernommen werden? Welche medizinischen Tätigkeiten müssen zwingend von der Ärztin oder vom Arzt ausgeführt werden?

- Haftung und Verantwortung: Wer haftet für fehlerhafte Modelle, z.B. wenn ein Modell nach einer allfälligen Lernanpassung mit neuen Patientendaten fehlerhafte Ergebnisse generiert?

\section{Sicht der Studierenden}

Sowohl an der Universität Bern als auch an der Universität Luzern wurden vom Studiendekan mit jeweils fünf Vertreterinnen oder Vertretern der Studierenden, die von den lokalen Fachvereinen der Medizinstudierenden angefragt wurden, Diskussionen zur Bedeutung des Themas Digitalisierung in der Medizin durchgeführt. Die Diskussionen fanden online statt und 
dauerten jeweils eine Stunde. Die Studierenden wurden nicht nach dem Zufallsprinzip ausgewählt, und damit sind die Ergebnisse der Diskussion zwar nicht repräsentativ, aber ergaben doch klare Hinweise auf relevante Themen, die in der Ausbildung berücksichtigt werden sollten.

Die Studierenden brachten klar zum Ausdruck, dass das Thema «Digitalisierung der Medizin» für sie relevant ist und in die Ausbildung integriert werden sollte.

\section{Medizinstudierende wollen auch etwas über die ethischen und rechtlichen Aspekte der Digitalisierung lernen.}

Als Themen der Digitalisierung, die aus ihrer Sicht wichtig sind, nannten sie Telemedizin, mobile Gesundheitsapps, Künstliche Intelligenz und maschinelles Lernen.

Sie wünschen sich, dass im Unterricht von der klinischen Anwendung digitaler Methoden ausgegangen wird und die theoretischen Grundlagen im Kontext der klinischen Anwendung gelehrt werden. In verschiedenen Spitälern sind bereits digitale Techniken im Einsatz, und diese wären nach Meinung der Studierenden interessante Ausgangspunkte für den Unterricht.

Bezüglich Künstlicher Intelligenz und maschinellen Lernens erachten es die Studierenden als sehr sinnvoll,

\section{Das Wichtigste in Kürze}

- Maschinelles Lernen wird zunehmend zum Einsatz kommen in der Medizin. Es ist deshalb wichtig, dass Ärztinnen und Ärzte ein Grundverständnis dieserTechnologien haben.

- Die Arbeitsgruppe "Digitalisierung der Medizin» des Bildungsnetzwerkes Medizin hat erörtert, welche Implikationen die Digitalisierung auf die Medizin hat und welche Bildungsinhalte zum Thema maschinelles Lernen den Medizinstudierenden vermittelt werden sollten.

- Sie schlägt folgende Lernziele vor: Basiswissen über Daten und Datenverwaltung, Grundwissen zu Konzepten, Validierung von Ergebnissen, die Ärztinnen und Ärzte mit maschinellem Lernen erzeugen, Wissen über die Anwendung in der medizinischen Praxis und Kenntnisse über rechtliche und ethische Aspekte dieser Anwendung. dass sie im Studium ein Grundverständnis darüber erwerben und insbesondere lernen, wie die Ergebnisse beurteilt (validiert) und interpretiert werden können. Die zu einer klinischen Tätigkeit neigenden Studierenden sehen die Validierung als sehr relevant und prioritär an. Studierende, die eine wissenschaftliche Tätigkeit planen, möchten mehr als nur die Konzepte und Methoden verstehen; sie möchten in Zukunft diese Methoden auch anwenden können.

Die Studierenden wollen auch etwas über die ethischen und rechtlichen Aspekte der Digitalisierung lernen und etwas über den potenziellen Nutzen und die Risiken erfahren. Zudem möchten sie über die möglichen Veränderungen informiert werden, die die Digitalisierung auf ihre spätere ärztliche Tätigkeit haben könnte. Die Studierenden äusserten ausserdem den Wunsch, dass definiert und auch in PROFILES abgebildet wird, welches Wissen und welche Fähigkeiten sie am Ende des Studiums beherrschen sollten.

Die Teilnehmenden des Workshops danken Aline Tannò (School of Medicine, HSG) für die Verfassung des Protokolls und die Aufzeich nung der Gespräche und den Studierenden der Universitäten Bern und Luzern für die Teilnahme an der Diskussion.

\section{Bildnachweis}

Monkey Business Images | Dreamstime.com

Glenn Carstens-Peters / Unsplash

\section{L'essentiel en bref}

- L'apprentissage automatique sera de plus en plus utilisé en médecine. Il est donc important que les médecins aient une compréhension de base de ces technologies.

- Le groupe de travail «Numérisation de la médecine» du réseau de formation en médecine a discuté des implications de la numérisation pour la médecine et des contenus de formation sur l'apprentissage automatique qui devraient être enseignés aux étudiants en médecine.

- Le groupe propose les objectifs d'apprentissage suivants: connaissances de base sur les données et la gestion des données, connaissances de base sur les concepts, validation des résultats que les médecins produisent avec l'apprentissage automatique, connaître l'application dans la pratique médicale ainsi que les aspects juridiques et éthiques de cette application. 